\title{
CONVERGENCE RATE OF MONOTONE NUMERICAL SCHEMES FOR HAMILTON-JACOBI EQUATIONS WITH WEAK BOUNDARY CONDITIONS
}

\author{
KNUT WAAGAN*
}

\begin{abstract}
We study a class of monotone numerical schemes for time-dependent HamiltonJacobi equations with weak Dirichlet boundary conditions. We get a convergence rate of $\frac{1}{2}$ under some usual assumptions on the data, plus an extra assumption on the Hamiltonian $H(D u, x)$ at the boundary $\partial \Omega$. More specifically the mapping $p \rightarrow H(p, x)$ must satisfy a monotonicity condition for all $p$ in a certain subset of $\mathbf{R}^{n}$ given by $\Omega$. This condition allows the use of the interior subsolution conditions at the boundary in the comparison arguments. We also prove a comparison result and Lipschitz regularity of the exact solution. As an example we construct a Godunov type scheme that can handle the weakened boundary conditions.
\end{abstract}

Key words. Hamilton-Jacobi equations, viscosity solutions, weak boundary conditions, numerical approximation

AMS subject classifications. 35F $30,49 \mathrm{~L} 25,65 \mathrm{M} 12$

1. Introduction. Let the scalar function $u(x, t)$ solve the Hamilton-Jacobi equation

$$
u_{t}+H(D u, x)=0
$$

for $x$ in an open and bounded set $\Omega \in \mathbf{R}^{n}$, and $t$ in a bounded interval $(0, T)$. We will show well-posedness, Lipschitz regularity, and study numerical approximations to this equation under the Cauchy-Dirichlet boundary condition

$$
\begin{aligned}
& u(x, 0)=u_{0}(x), \quad x \in \bar{\Omega} \\
& u(x, t)=g(x), \quad x \in \partial \Omega, 0 \leq t<T .
\end{aligned}
$$

Note that $g=\left.u_{0}\right|_{\partial \Omega}$. The mapping $p \rightarrow H(p, x)$ is assumed to tend uniformly to $+\infty$ as $|p| \rightarrow \infty$, and to do so in a later specified monotone way. In addition, some Lipschitz estimates on the data and some geometric conditions on the domain will be prescribed.

Classical solutions to (1.1) may develop discontinuities in the first derivative in finite time, hence viscosity solutions, as defined in [11], are more natural to study, since they are only continuous. Overviews of this subject are found in [10], [9] and [2]. The term 'viscosity solution' comes from the 'vanishing viscosity method' where a solution to the first order equation is sought by solving $u_{t}+H(D u, x)=\epsilon \Delta u$, and letting $\epsilon \rightarrow 0$, typically resulting in a viscosity solution. The viscosity solution concept also ensures 'causality' in the sense that characteristics are not allowed to emanate from the singularities in $D u$. These facts reveal a connection to the entropy solution of scalar conservation laws which is more than superficial, but the two theories are independent. However, numerical schemes for Hamilton-Jacobi equations are often derived from numerical schemes for conservation laws.

Since characteristics may be directed out of the boundary, the Cauchy-Dirichlet problem may be overdetermined both classically and in the original viscosity solutions setting, hence a weakened boundary condition is called for. The papers [5] and

${ }^{*}$ Center of Mathematics for Applications, P.O. Box 1053 Blindern, NO-0316 Oslo, NORWAY. Email: knut.waagan@cma.uio.no. Completed while at the Department of Applied Mathematics, Würzburg University, Germany. 
[14] both describe what has turned out to be the natural way to perform such a generalization, since the consistency with both control theory and vanishing viscosity approximation is maintained. This theory also includes other types of boundary data, as seen in for example [14] and [10], with Neumann conditions as another special case.

Before continuing, we need to specify what we mean by a viscosity solution. First, we state that by $C^{1}(\bar{\Omega} \times[0, T])$ we mean the class of all functions $\psi$ such that $\psi$ is a restriction to $\bar{\Omega} \times[0, T]$ of a function in $C^{1}\left(R^{n} \times R\right)$.

We define a viscosity solution to (1.1)-(1.2) as follows.

Definition 1. Let $\psi \in C^{1}(\bar{\Omega} \times[0, T])$. A function $u(x, t)$ is a subsolution of (1.1)-(1.2) if whenever $u^{*}-\psi$ has a local maximum at $(x, t) \in \bar{\Omega} \times[0, T]$, then

$$
\begin{aligned}
\psi_{t}(x, t)+H(D \psi(x, t), x) & \leq 0, \quad x \in \Omega, 0<t \leq T \\
\min \left(u^{*}(x, t)-g(x), \psi_{t}(x, t)+H(D \psi(x, t), x)\right) & \leq 0, \quad x \in \partial \Omega, t>0 \\
u^{*}(x, 0) & \leq u_{0}(x), \quad x \in \bar{\Omega} .
\end{aligned}
$$

A function $v$ is a supersolution of (1.1)-(1.2) if whenever $v_{*}-\psi$ has a local minimum at $(x, t) \in \bar{\Omega} \times[0, T]$, then

$$
\begin{aligned}
\psi_{t}(x, t)+H(D \psi(x, t), x) & \geq 0, \quad x \in \Omega, 0<t \leq T \\
\max \left(v_{*}(x, t)-g(x), \psi_{t}(x, t)+H(D \psi(x, t), x)\right) & \geq 0, \quad x \in \partial \Omega, t>0 \\
v_{*}(x, 0) & \geq u_{0}(x), \quad x \in \bar{\Omega} .
\end{aligned}
$$

A function $u$ is a viscosity solution of (1.1)-(1.2) if it is both a sub- and a supersolution.

The notations $\cdot^{*}$ and $\cdot_{*}$ refer to the usual upper and lower semicontinuous envelope, that is

$$
u^{*}(x, t)=\limsup _{y \in \bar{\Omega} \rightarrow x, s \in[0, T] \rightarrow t} u(y, s), \quad \text { and } \quad u_{*}(x, t)=\liminf _{y \in \bar{\Omega} \rightarrow x, s \in[0, T] \rightarrow t} u(y, s) .
$$

We have chosen to simply include the endpoint $t=T$ in our definition, but leaving the time interval open would have been equivalent. This is due to the monotone dependence on $u_{t}$ in (1.1), which also makes weakening the initial condition redundant (See $[5])$.

The uniqueness of viscosity solutions follows if one can prove that $u \leq v$ for all subsolutions $u$ and supersolutions $v$. This is referred to as a comparison result.

In [5] there is a comparison result for (1.1)-(1.2) under some common continuity assumptions on the data and a nondegeneracy condition at the boundary. This nondegeneracy condition has uniform coercivity of $H$ as a special case. We prefer to give a new comparison proof here since the proof in [5] requires uniform continuity of $p \rightarrow H(p, x)$, which can be avoided in our setting.

In [7] a uniform coercivity condition on $p \rightarrow H(p, x)$, is shown to imply $\left.u^{*}\right|_{\partial \Omega} \leq g$, and a comparison result for subsolutions that are lower Lipschitz bounded in time. The authors then construct a solution where this holds for initial data such that $H\left(D u_{0}, \cdot\right) \leq 0$ using vanishing viscosity arguments. They also prove Lipschitz continuity of subsolutions that are nondecreasing in time under assumptions which are no stronger than will be made here.

The solution given by Definition 1 is the optimal value function for a finite horizon control problem where the cost stops running once a trajectory reaches $\partial \Omega$. This problem is covered in [13]. When the boundary condition is only weakly satisfied at $x \in \partial \Omega$, it is because the optimal trajectory starting at $x$ does not exit $\Omega$ immediately. 
The uniform convergence of monotone approximation schemes for the Cauchy problem was studied in [8] and [17]. The solution remains Lipschitz continuous if it is initially, and the convergence rate is $\frac{1}{2}$. In [6], locally uniform convergence for quite general boundary value problems is proved, also for second order degenerate elliptic cases. The main condition in the proof is that the approximated problem satisfies a comparison principle. No explicit error estimate is given, and since schemes that form boundary layers are allowed, a uniform convergence rate would not be possible. Putting these results into practice for first order equations is the subject of [1]. Triangular grids are considered, and a Godunov scheme is extended to the boundary by reinterpreting the Hamiltonian as the Legendre transform of a control problem. Furthermore a Lax-Friedrichs scheme is also extended to the boundary, and certain nonconvex cases are also treated. Uniform coercivity of the Hamiltonian is shown to imply Lipschitz continuity of approximations to the stationary equation. No control theory will be used in this article, but our extension of the Godunov scheme to the boundary is still very similar to that of [1].

Our main result is an estimate of $\frac{1}{2}$ for the convergence rate of monotone numerical schemes for problem (1.1)-(1.2). To achieve this result, we add conditions on $H$ and $\Omega$. Roughly speaking, we will require that as long as $p \cdot n<0$ for the inward normal vector $n$, then $p \rightarrow H(p, x)$ is nondecreasing in the direction of $-n$. Special cases included are radially symmetric $H$ on convex $\Omega$, and $H$ given by $H\left(u_{x_{1}}^{2}, \ldots, u_{x_{n}}^{2}\right)$, where $H$ is nondecreasing in each argument, and $\Omega$ is a rectangle oriented like $(0,1)^{n}$. The proof is built on the treatment of the Cauchy problem in [8] and [17].

After stating some assumptions and preliminary results, we will specify the requirements at the boundary. Next a comparison result is given, from which existence and regularity follows. A generic numerical scheme is then defined, for which we state the main result on convergence rate. This is formulated in the tradition of [8] and [17], with the necessary extensions made to include the boundary conditions.

Finally we formulate a Godunov type scheme as an example. This turns out to have some theoretical difficulties other than just stating what happens at the grid points, since how to interpolate between the boundary and interior grid points is generally not obvious. Hence only stating what happens at the grid points is insufficient in this setting, although it may make sense for the Cauchy problem. The simple numerical boundary condition $u(x, t)=g(x)$, is not included in the analysis. Of course, we would not generally expect a uniform estimate for such a scheme since boundary layers could form, as they do for vanishing viscosity approximations. Our numerical results demonstrate convergence properties that are similar to what is known for pure Cauchy problems, as our convergence estimate predicts.

2. Assumptions and preliminary results. This section sums up some assumptions, definitions and results we will need. The $L^{\infty}$-norm of a function $f$ is denoted $\|f\|$, and hence, $\|D f\|$ denotes the Lipschitz seminorm of $f$, since a Lipschitz continuous function is differentiable almost everywhere by Rademacher's theorem.

We will make the following Lipschitz assumptions:

Assumption 1. The mapping $p \rightarrow H(p, x)$ is continuous, and for any $R>0$, there exists $C_{R}>0$ such that

$$
|H(p, x)-H(p, y)| \leq C_{R}|x-y|, \quad \forall x, y \in \bar{\Omega},|p| \leq R .
$$

The initial value $u_{0}$ is Lipschitz continuous, so one may define

$$
L_{0}=\max \left(\left\|D u_{0}\right\|, p_{0}\right), \quad \text { where } p_{0}=\sup _{x \in \Omega}\{|p|: H(p, x)=0\}<\infty .
$$


By uniform coercivity, we mean, as in [3], [4] and [7]:

Assumption 2. The mapping $p \rightarrow H(p, x)$ is uniformly coercive, that is for any $C \in \mathbf{R}$ there exists $r>0$ such that $H(p, x)>C$ for all $|p|>r$ and $x \in \bar{\Omega}$.

This is used to get Lipschitz continuity of $u$, and it also gives the following proposition. It requires yet another assumption, this one on $\Omega$, which we state first. It is an exterior sphere condition given locally.

Assumption 3. For each $x \in \partial \Omega$, there exist $r_{x}>0$ and $z_{x} \in \mathbf{R}^{n}$ defining an open ball $B\left(z_{x}, r_{x}\right)$, such that $\bar{B}\left(z_{x}, r_{x}\right) \cap \bar{\Omega}=\{x\}$.

Proposition 2.1. If Assumptions 2 and 3 hold, then

$$
u^{*}(x, t) \leq g(x), \quad \forall x \in \partial \Omega, t \in[0, T]
$$

holds for any subsolution $u$ of (1.1)-(1.2) that is bounded above.

Our proof essentially copies the one in [7], which treated $C^{1}$ boundaries. A more intuitive motivation for this result is the fact that superdifferentials become unbounded on $\partial \Omega$.

Proof. Assume in order to obtain a contradiction that there exists $\left(x_{0}, t_{0}\right) \in$ $\partial \Omega \times(0, T]$ such that $u^{*}\left(x_{0}, t_{0}\right)>g\left(x_{0}\right)$. Consider

$$
\Psi(x, t)=u^{*}(x, t)-\frac{\left(t-t_{0}\right)^{2}}{2 \beta^{2}}-\frac{1}{\alpha}\left(\left|x-z_{x_{0}}\right|-\left|x_{0}-z_{x_{0}}\right|\right), \quad \alpha, \beta>0 .
$$

The point $z_{x_{0}}$ is given by Assumption 3, hence the last term of $\Psi$ is nonnegative. Denote the maximum point of $\Psi$ in $\bar{\Omega} \times[0, T]$ by $(\hat{x}, \hat{t})$. Now,

$$
u^{*}\left(x_{0}, t_{0}\right)=\Psi\left(x_{0}, t_{0}\right) \leq \Psi(\hat{x}, \hat{t}) \leq u^{*}(\hat{x}, \hat{t})-C\left(\left|\hat{x}-z_{x_{0}}\right|-\left|x_{0}-z_{x_{0}}\right|\right)-\frac{\left(\hat{t}-t_{0}\right)^{2}}{2 \beta^{2}} .
$$

Since $u^{*}$ is bounded by, say, $R>0$, this means that $\left(\left|\hat{x}-z_{x_{0}}\right|-\left|x_{0}-z_{x_{0}}\right|\right) \leq 2 R \alpha$ and $\left|\hat{t}-t_{0}\right| \leq 2 \sqrt{R} \beta$. This implies $u^{*}(\hat{x}, \hat{t}) \rightarrow u^{*}\left(x_{0}, t_{0}\right)$ as $\alpha$ and $\beta$ go to zero, hence, taking $\alpha$ and $\beta$ small enough, we have $u^{*}(\hat{x}, \hat{t})>g(\hat{x})$. That means we may use the differential equation, so

$$
\frac{\hat{t}-t_{0}}{\beta^{2}}+H\left(\frac{\hat{x}-z_{x_{0}}}{\alpha\left|\hat{x}-z_{x_{0}}\right|}, \hat{x}\right) \leq 0 .
$$

By uniform coercivity of $H$, this implies

$$
\frac{1}{\alpha} \leq \Gamma_{\beta}
$$

for some $\Gamma_{\beta}<\infty$. So choosing $\alpha<\frac{1}{\Gamma_{\beta}}$ produces the contradiction.

2.1. Subsolutions on the boundary. In this section we state the monotonicity requirement of $H$ at $\partial \Omega$. The aim is to give conditions such that the interior subsolution condition holds on all $\bar{\Omega}$, at least for test functions of the form typically used in a doubling of variables argument. The key observation is that the gradient of such a test function points out of the domain for certain choices of $H$ and $\Omega$. Hence, monotonicity of $p \rightarrow H(p, x)$ only needs to hold for $p$ in a subset of $\mathbf{R}^{n}$. This is similar to the results of [12] and [16] with the exception that these require global monotonicity of $H$. In the simplest case that $\partial \Omega$ is $C^{1}$ and convex, with inward normal vector $n(x)$, we need

$$
H(k(x-z)-\mu n(x), x) \geq H(k(x-z), x), \quad \forall k, \mu>0, x \in \partial \Omega, z \in \bar{\Omega} .
$$


However, to allow corners, we require that

Assumption 4. There exists a nonnegative function $d \in C^{1}(\Omega) \cap C^{0}(\bar{\Omega})$ such that $d(x)=0$ if and only if $x \in \partial \Omega$. Furthermore, for every $x_{0} \in \partial \Omega, k>0$, positive function $\mu: \Omega \rightarrow \mathbf{R}$, and $z \in \bar{\Omega}$,

$$
\liminf _{x \rightarrow x_{0}} H(k(x-z)-\mu(x) D d(x), x) \geq H\left(k\left(x_{0}-z\right), x_{0}\right) .
$$

We cannot simply state the monotonicity property at the point $x_{0}$, since $\operatorname{Dd}\left(x_{0}\right)$ will be 0 at any corner, and because we need to allow $\mu(x)$ to go to infinity at $\partial \Omega$. Note, however that where $\partial \Omega$ is $C^{1}, D d(x)$ must be parallel to the inward normal vector $n(x)$, hence $(2.8)$ suffices.

We could have reduced the domain of $z$ by restricting $\left|z-x_{0}\right|$, but that would be a bit technically complicated to use in the proof of convergence rate. The assumption becomes useful through the following lemma:

Lemma 2.2. Let Assumption 4 hold. Let u be a bounded subsolution of (1.1)-(1.2) such that

$$
u^{*}(x, t)=\limsup _{y \in \Omega \rightarrow x, s \in(0, T) \rightarrow t} u(y, s) .
$$

Suppose the mapping

$$
(x, t) \rightarrow u^{*}(x, t)-\frac{k}{2}(x-z)^{2}-\eta(t)
$$

attains its maximum over $\bar{\Omega} \times[0, T]$ at $\left(x_{0}, t_{0}\right)$ with $x_{0} \in \partial \Omega, t_{0}>0, k>0, z \in \bar{\Omega}$ and $\eta \in C^{1}((0, T))$. Then

$$
\eta_{t}\left(t_{0}\right)+H\left(k\left(x_{0}-z\right), x_{0}\right) \leq 0
$$

Proof. Without losing generality, we may assume the maximum is strict. Consider

$$
\Psi(x, t)=u^{*}(x, t)-\frac{k}{2}(x-z)^{2}-\eta(t)-\frac{\epsilon}{d(x)} .
$$

Because of (2.10), $\Psi$ will have a local maximum $\left(x_{\epsilon}, t_{\epsilon}\right)$ with $x_{\epsilon} \in \Omega$, such that $\left(x_{\epsilon}, t_{\epsilon}\right) \rightarrow\left(x_{0}, t_{0}\right)$ as $\epsilon$ goes to 0 . Hence

$$
0 \geq \eta_{t}\left(t_{\epsilon}\right)+H\left(k\left(x_{\epsilon}-z\right)-\epsilon \frac{D d\left(x_{\epsilon}\right)}{d\left(x_{\epsilon}\right)^{2}}, x_{\epsilon}\right) .
$$

Letting $\epsilon \rightarrow 0$, the Lemma follows from (4).

2.1.1. Examples. An example where Assumption 4 holds is if $p \rightarrow H(p, x)$ is radially symmetric with a unique minimum at $p=0$ (i.e. $H(p, x)=h(|p|, x)$ where $h:[0, \infty) \times \mathbf{R}^{n} \rightarrow \mathbf{R}$ is nondecreasing in the first variable), $\Omega$ is convex and $\partial \Omega$ is piecewise $C^{1}$. The function $d$ can be chosen as the product of the distances to each $C^{1}$ subset of $\partial \Omega$.

As a second example consider $\Omega=(0,1)^{n}$. Then the assumption holds for any Hamiltonian $H(p, x)=H_{2}\left(p_{1}^{2}, p_{2}^{2}, \ldots, p_{n}^{2}, x\right)$ if $p_{i}^{2} \rightarrow H_{2}$ is nondecreasing for all $1 \leq$ $i \leq n$. We can choose $d\left(x_{1}, \cdots, x_{n}\right)=\Pi_{1}^{n} x_{i}\left(1-x_{i}\right)$. 
An example where the Lemma fails is if $H=H(p): \mathbf{R}^{2} \rightarrow \mathbf{R}$ has level sets that are ellipses with no axis parallel to the normal vector $D d$. To demonstrate this we consider for simplicity $\Omega=\{x=(\xi, \eta): \xi>0\} \in \mathbf{R}^{2}$ (hence $d(x)=\xi$ is a reasonable choice of $d$ ), and take

$$
H(D u)=\hat{H}\left(u_{\xi}, u_{\eta}\right)=\left(u_{\xi}-u_{\eta}\right)^{2}+\frac{1}{4}\left(u_{\xi}+u_{\eta}\right)^{2}-1 .
$$

For instance, we may choose $k, x_{0}$ and $z$ in Lemma 2.2 such that $k\left(x_{0}-z\right)=(0,-1)$, and get

$$
H\left(k\left(x_{0}-z\right)\right)=\hat{H}(0,-1)>\hat{H}(-\mu,-1)=H\left(k\left(x_{0}-z\right)-\mu D d\left(x_{0}\right)\right)
$$

for all $\mu \in\left(0, \frac{6}{5}\right)$. Hence Assumption 4 fails at the line $\xi=0$ for $H=\hat{H}$.

2.1.2. Translation of the zero gradient. In Assumption 4, the value $p=0$ is always included in the region of $\mathbf{R}^{n}$ where $p \rightarrow H(p, x)$ has to be monotone along $D d$, but such a restriction may be remedied by a translation; Consider the situation that Assumption 4 holds for the Hamiltonian $p \rightarrow H(p-m(x), x)$, and that there exists a differentiable $f$ such that $D f=m$. Then $w(x, t)=u(x, t)+f(x)$ will solve

$$
\begin{aligned}
w_{t}+H(D w-m(x), x) & =0, \quad w(x, 0)=u_{0}(x)+f(x) \quad \forall x \in \Omega \\
w(x, t) & =g(x)+f(x) \quad \forall x \in \partial \Omega
\end{aligned}
$$

in the viscosity sense.

As a special case consider $\Omega=(0,1) \in \mathbf{R}$. Then, according to the above, $H(p, 0)$ must be decreasing for $p<a$ and $H(p, 1)$ must be increasing for $p>b$ for some constants $a$ and $b$.

3. Uniqueness, existence and regularity. In this section we prove the following:

ThEOREM 3.1. Let assumptions 1 - 4 hold, then there exists one and only one viscosity solution $u$ of (1.1)-(1.2) in $C^{0}(\bar{\Omega} \times[0, T])$. Furthermore

$$
\left\|u_{t}\right\| \leq L_{t}, \text { and }\|D u\| \leq L,
$$

where

$$
L_{t}=\sup _{|p| \leq L_{0}, x \in \Omega}|H(p, x)|,
$$

and $L$ is any number such that $H(p, x)>L_{t}$ for all $|p|>L$, and $x \in \Omega$.

Note that $L \geq L_{0}$, and that $L$ may be finite because of the uniform coercivity condition.

We first state that it is enough to prove the theorem for problem (1.1)-(1.2) with a truncated Hamiltonian, more specifically the problem

$$
\begin{aligned}
u_{t}+H_{R}(D u, x) & =0, \quad \forall x \in \Omega, t \in(0, T), \\
u(x, 0) & =u_{0}(x), \quad x \in \bar{\Omega}, \\
u(x, t) & =g(x), \quad x \in \partial \Omega, 0 \leq t<T,
\end{aligned}
$$

where $H_{R}(p, x)=\min (H(p, x), R)$, for some finite $R>L_{t}$. The advantage is that $H_{R}$ is globally Lipschitz in the $x$-variable. Clearly the solution of (3.2) also solves the 
original problem (1.1)-(1.2) because of (3.1). The uniqueness statement of Theorem 3.1 follows since we also show that comparison holds for (1.1)-(1.2) provided either the subsolution or the supersolution is Lipschitz continuous. The Hamiltonian $H_{R}$ satisfies Assumption 4, but not Assumption 2, and hence Proposition 2 does not apply. However it is enough to prove Theorem 3.1 for the problem (3.2) under the explicit assumption that the solution $u$ of (3.2) satisfies $\left.u\right|_{\partial \Omega} \leq g$.

The crucial point is the following comparison result.

TheOrem 3.2. Assume assumptions 1 and 4 hold, and that one of the following assumptions holds:

1. Let $u$ be a bounded subsolution, and $v$ a bounded supersolution of (3.2). Let $u$ satisfy (2.10), and let $\left.u^{*}\right|_{\partial \Omega} \leq g$.

2. Let $u$ be a bounded subsolution, and $v$ a bounded supersolution of (1.1)-(1.2). Let $u$ satisfy (2.10), and assume either $u$ or $v$ is Lipschitz continuous.

Then

$$
u^{*} \leq v_{*}
$$

in $\bar{\Omega} \times[0, T]$.

Note that provided $\left.u^{*}\right|_{\partial \Omega} \leq g$, the function $\lim \sup _{y \in \Omega \rightarrow x, s \in(0, T) \rightarrow t} u(y, s)$ is a subsolution. Hence the comparison result implies that, given a viscosity solution $u$ of (3.2) such that $\left.u^{*}\right|_{\partial \Omega} \leq g$, we have that $u_{*}$ is in $C^{0}(\bar{\Omega} \times[0, T])$, and is a viscosity solution of (3.2). However, $u$ can be discontinuous at the boundary in general.

The proof is standard due to Lemma 2.2, the only new problem is handling the boundary condition for the supersolution, which can be done quite easily.

Proof. Define

$$
M=\sup \left(u^{*}(x, t)-v_{*}(x, t)-2 \delta t\right)
$$

We assume $M>0$, and proceed to show that this leads to a contradiction for any constant $\delta>0$. Let

$$
\Psi(x, t, y, s)=u^{*}(x, t)-v_{*}(y, s)-\delta(t+s)-\frac{(x-y)^{2}}{2 \epsilon^{2}}-\frac{(t-s)^{2}}{2 \epsilon^{2}} .
$$

Let

$$
\left(x_{\epsilon}, t_{\epsilon}, y_{\epsilon}, s_{\epsilon}\right) \in(\bar{\Omega} \times[0, T])^{2}
$$

be a maximum point of $\Psi$ in $(\bar{\Omega} \times[0, T])^{2}$.

Then,

$$
\begin{aligned}
u^{*}\left(x_{\epsilon}, t_{\epsilon}\right) & -v_{*}\left(y_{\epsilon}, s_{\epsilon}\right)-\delta\left(t_{\epsilon}+s_{\epsilon}\right)-\frac{\left(x_{\epsilon}-y_{\epsilon}\right)^{2}}{2 \epsilon^{2}}-\frac{\left(t_{\epsilon}-s_{\epsilon}\right)^{2}}{2 \epsilon^{2}} \\
& =\Psi\left(x_{\epsilon}, t_{\epsilon}, y_{\epsilon}, s_{\epsilon}\right) \geq \Psi(x, t, x, t) \geq u^{*}(x, t)-v_{*}(x, t)-2 \delta t .
\end{aligned}
$$

Taking the supremum of the last expression, we see that

$$
u^{*}\left(x_{\epsilon}, t_{\epsilon}\right)-v_{*}\left(y_{\epsilon}, s_{\epsilon}\right)-\delta\left(t_{\epsilon}+s_{\epsilon}\right) \geq M,
$$

and by standard arguments (see for example [2]), and taking a subsequence if necessary, we also get

$$
x_{\epsilon} \rightarrow x^{*}, y_{\epsilon} \rightarrow x^{*}, \quad t_{\epsilon} \rightarrow t^{*}, s_{\epsilon} \rightarrow t^{*}
$$


where the supremum in (3.4) is obtained at $\left(x^{*}, t^{*}\right)$. If $x^{*} \in \Omega$, or if $t^{*}=0$, the proof is similar to the standard case.

If $x^{*} \in \partial \Omega$, we need to consider the possibilities that $x_{\epsilon}$ or $y_{\epsilon}$ are in $\partial \Omega$ as $\epsilon \rightarrow 0$. The case $x_{\epsilon} \in \partial \Omega$ can be treated in the same way as if $x_{\epsilon} \in \Omega$ due to Lemma 2.2. If $y_{\epsilon} \in \partial \Omega$, there are essentially two cases. First, if there are arbitrarily small $\epsilon$ such that $v_{*}\left(y_{\epsilon}, s_{\epsilon}\right)<g\left(y_{\epsilon}\right)$, we may argue as above. Otherwise, we may consider $\epsilon$ such that $v_{*}\left(y_{\epsilon}, s_{\epsilon}\right) \geq g\left(y_{\epsilon}\right)$, in which case (3.8) implies

$$
\begin{aligned}
M & \leq u^{*}\left(x_{\epsilon}, t_{\epsilon}\right)-v_{*}\left(y_{\epsilon}, s_{\epsilon}\right) \\
& \leq u^{*}\left(x_{\epsilon}, t_{\epsilon}\right)-g\left(y_{\epsilon}\right), \quad y_{\epsilon} \in \partial \Omega .
\end{aligned}
$$

Then, by $(3.9)$ and $\left.u^{*}\right|_{\partial \Omega} \leq g$,

$$
M \leq \limsup _{\epsilon \rightarrow 0}\left\{u^{*}\left(x_{\epsilon}, t_{\epsilon}\right)-u_{0}\left(y_{\epsilon}\right)\right\} \leq u^{*}\left(x^{*}, t^{*}\right)-u_{0}\left(x^{*}\right) \leq 0 .
$$

We can prove the existence of a viscosity solution such that $\left.u\right|_{\partial \Omega} \leq g$ by a standard application of Perron's method as adapted to viscosity solutions, see for example [2]. Note that as a supersolution we can take $u_{0}(x)+2 L \operatorname{dist}(x)$, where $\operatorname{dist}(x)$ is the distance function to $\partial \Omega$, and as a subsolution we can take $u_{0}(x)-L_{t} t$.

From the comparison result we easily get

Proposition 3.3. Let the assumptions 1 and 4 hold, and let $u \in C^{0}(\bar{\Omega} \times[0, T])$ be a viscosity solution of (3.2) such that $\left.u\right|_{\partial \Omega} \leq g$. Then

$$
\|u(\cdot, t)\| \leq\left\|u_{0}\right\|+C_{0} t, \quad C_{0}=\max _{x \in \bar{\Omega}}|H(0, x)|
$$

and

$$
\left|u(x, t)-u_{0}(x)\right| \leq L_{t} t
$$

Proof. This follows from $\left\|u_{0}\right\|+C_{0} t$ and $u_{0}(x)+L_{t} t$ being supersolutions, and - $\left\|u_{0}\right\|-C_{0} t$ and $u_{0}(x)-L_{t} t$ being subsolutions.

We now turn to Lipschitz regularity.

THEOREM 3.4. Let the assumptions 1 and 4 hold, and let $u \in C^{0}(\bar{\Omega} \times[0, T])$ be a viscosity solution of $(3.2)$ such that $\left.u\right|_{\partial \Omega} \leq g$. Then

$$
u(x, t+\tau)-u(x, t) \leq L_{t} \tau
$$

for $\tau>0$.

Proof. This follows from

$$
\tilde{u}(x, t)=u(x, t+\tau)-L_{t} \tau
$$

being a subsolution. This is easily seen for $t>0$ and $x \in \Omega$, and by Proposition 3.3,

$$
\tilde{u}(x, 0)=u(x, \tau)-L_{t} \tau \leq u_{0}(x) .
$$

Finally, at the boundary points $x \in \partial \Omega$, we have $\tilde{u}(x, t) \leq u(x, t) \leq g(x)$.

The lower bound on $u_{t}$ follows similarly by

$$
(x, t) \mapsto u(x, t+\tau)+L_{t} \tau
$$


being a subsolution. A coercivity argument could also have been used for the upper bound.

The Lipschitz continuity in $x$ follows from Assumption 2, since it implies that

$$
\underline{u}(x, t)=\sup _{y \in \bar{\Omega}}\{u(y, t)-k|y-x|\}
$$

is a subsolution whenever $k>L$ with $L$ defined as in Theorem 3.1: Consider first $t=0$. Clearly there exists $y_{0}$ such that

$$
u_{0}(y)-k|x-y| \leq \underline{u}(x, 0)=u_{0}\left(y_{0}\right)-k\left|x-y_{0}\right| .
$$

More specifically $u_{0}(x) \leq u_{0}\left(y_{0}\right)-k\left|x-y_{0}\right|$, hence, unless $y_{0}=x$, we get the contradiction $k \leq L_{0} \leq L$, so $\underline{u}(x, 0)=u_{0}(x)$. Next, consider $\underline{u}(x, t)-\psi(x, t)$ having a maximum point $\left(x_{0}, t_{0}\right)$ with $t_{0}>0$. Then there exists $y_{0}$ such that

$$
u(y, t)-k|y-x|-\psi(x, t) \leq u\left(y_{0}, t_{0}\right)-k\left|y_{0}-x_{0}\right|-\psi\left(x_{0}, t_{0}\right)
$$

If $x_{0} \neq y_{0}$, we get

$$
\psi_{t}\left(x_{0}, t_{0}\right)+H_{R}\left(k \frac{x_{0}-y_{0}}{\left|x_{0}-y_{0}\right|}, y_{0}\right) \leq 0,
$$

but by the Lipschitz continuity in time $\left|\psi_{t}\left(x_{0}, t_{0}\right)\right| \leq L_{t}$, which contradicts $k>L$. Hence $x_{0}=y_{0}$, so, by (3.19),

$$
\psi_{t}\left(x_{0}, t_{0}\right)+H_{R}\left(D \psi\left(x_{0}, t_{0}\right), x_{0}\right) \leq 0 .
$$

Because of Lemma 2.2, this also holds if $y_{0} \in \partial \Omega$.

4. The convergence result. The convergence result may now be stated. We consider a scheme $S^{\lambda}(\rho): B_{g} \rightarrow B_{g}$, where $B_{g}$ is a class of functions in $L^{\infty}(\bar{\Omega})$ such that all Lipschitz continuous functions $U$ satisfying $\left.U\right|_{\partial \Omega} \leq g$ are included in $B_{g}$. Furthermore $S^{\lambda}(\rho)$ is defined for all $\lambda>0$ sufficiently small, and $0 \leq \rho \leq \lambda$.

We define our approximate solution $U(x, t): \bar{\Omega} \times[0, T] \rightarrow \mathbf{R}$ inductively by setting

$$
U(x, 0)=u(x, 0), \quad x \in \bar{\Omega},
$$

and for $t \in\left(t_{n}, t_{n+1}\right)$, where $t_{n}=n \Delta t$ with $0 \leq \Delta t \leq \lambda$ :

$$
U(x, t)=S^{\lambda}\left(t-t_{n}\right) U\left(\cdot, t_{n}\right)(x), \quad x \in \bar{\Omega} .
$$

The time step $\Delta t$ must be chosen such that for $\rho<\Delta t, S^{\lambda}(\rho)$ satisfies the following conditions:

1.

$$
\left\|S^{\lambda}(\rho) U\right\| \leq\|U\|+\tilde{C}_{0} \rho
$$

for a constant $\tilde{C}_{0}$ independent of $\lambda$.

2 .

$$
\left\|S^{\lambda}(\rho) U-U\right\| \leq C_{S}(\|D U\|) \rho
$$

where $C_{S}(\cdot) \in C^{0}: \mathbf{R}_{0}^{+} \rightarrow \mathbf{R}_{0}^{+}$and independent of $\lambda$. 
3. Let $\psi(x)=\frac{k}{2}(x-z)^{2}+K$, and $\theta(x)=-\frac{k}{2}(x-z)^{2}+K$ for some $z \in \bar{\Omega}$ and constants $k, K \geq 0$. Then there is a $C>0$ such that the following holds for all $x \in \Omega$ : Given $U \in B_{g}$, we have either that

$$
\frac{\left(S^{\lambda}(\rho) \theta\right)_{*}(x)-\theta(x)}{\rho}+H(D \theta, x) \geq-C\left(1+\|D \theta\|+\left\|D^{2} \theta\right\|\right) \lambda
$$

whenever $\theta \leq U$, or that $(\xi, \tau) \rightarrow S^{\lambda}(\tau) U(\xi)$ is a supersolution of (1.1) in a neighborhood of $(x, \rho)$.

4. There exists an operator $S_{b}^{\lambda}: L^{\infty}(\bar{\Omega}) \rightarrow L^{\infty}(\bar{\Omega})$, such that for any $U \in B_{g}$, $S^{\lambda}(\rho) U \leq S_{b}^{\lambda}(\rho) U$, and

$$
S^{\lambda}(\rho) U(x)=\min \left(g(x), S_{b}^{\lambda}(\rho) U(x)\right) \quad \forall x \in \partial \Omega .
$$

Furthermore $S_{b}^{\lambda}$ satisfies

$$
\begin{gathered}
\frac{\left(S_{b}^{\lambda}(\rho) \theta\right)_{*}(x)-\theta(x)}{\rho}+H(D \theta(x), x) \geq-C\left(1+\|D \theta\|+\left\|D^{2} \theta\right\|\right) \lambda \\
\frac{\left(S_{b}^{\lambda}(\rho) \psi\right)^{*}(x)-\psi(x)}{\rho}+H(D \psi(x), x) \leq C\left(1+\|D \psi\|+\left\|D^{2} \psi\right\|\right) \lambda
\end{gathered}
$$

for all $x \in \bar{\Omega}$.

5. $S^{\lambda}$ and $S_{b}^{\lambda}$ are monotone in the sense that if $U_{1}(x) \leq U_{2}(x)$ for all $x \in \bar{\Omega}$, then

$$
S^{\lambda}(\rho) U_{1}(x) \leq S^{\lambda}(\rho) U_{2}(x) \quad \text { and } \quad S_{b}^{\lambda}(\rho) U_{1}(x) \leq S_{b}^{\lambda}(\rho) U_{2}(x)
$$

for all $x \in \bar{\Omega}$.

6. $S^{\lambda}$ is also monotone in $g$ in the following sense: For any function $\bar{g} \geq g$, there exists an operator $S_{\bar{g}}^{\lambda}: B_{\bar{g}} \rightarrow B_{\bar{g}}$ with $B_{g} \subseteq B_{\bar{g}}$ such that the above assumptions hold with $g$ replaced by $\bar{g}$, and

$$
S^{\lambda}(\rho) U \leq S_{\bar{g}}^{\lambda}(\rho) U
$$

for any $U \in B_{g}$.

7. There exists $L_{S} \geq L_{0}$ such that one of the following two conditions hold:

i) For all $y \in \partial \Omega$ and $k \geq L_{S}$,

$$
S_{\bar{g}}^{\lambda}(\rho)[g(y)+k|x-y|] \leq g(y)+k|x-y|,
$$

where $\bar{g}$ here denotes $k|x-y|+g(y)$ restricted to $\partial \Omega$.

ii) We have the Lipschitz estimate

$$
\left\|D S^{\lambda}(\rho) U\right\| \leq\|D U\|+L_{S}(1+\|D U\|) \rho .
$$

Here is our main result:

THEOREM 4.1. Let the assumptions 1-4 hold. Then $U(x, t)$ converges uniformly to the unique viscosity solution $u$ of (1.1)-(1.2) in $C^{0}(\bar{\Omega} \times[0, T])$, and

$$
|U(x, t)-u(x, t)| \leq K\left(\lambda^{\frac{1}{2}}+\lambda\right), \quad \forall(x, t) \in \bar{\Omega} \times[0, T]
$$

for a constant $K$ given by $\left\|u_{0}\right\|, C, C_{0}, \tilde{C}_{0}, C_{2 L}, C_{S}\left(L_{0}\right), L_{t}, L, L_{S}$ and $T$. 
Requiring both monotonicity and (4.7), means that schemes like $S^{\lambda}$ only exist for a certain class of Hamiltonians and domains. However, this class should coincide reasonably well with the one specified by Assumption 4, since $S_{b}^{\lambda}$ only needs to be consistent for gradients of the form $k(x-z)$ for which $H$ is monotone. Unfortunately, Lemma 2.2 does not translate directly to a corresponding lemma for approximations.

Before proving the theorem, we show how monotonicity and consistency of $S^{\lambda}$ imply that $U$ is in a sense almost a sub- and a supersolution (At least with respect to the test functions we consider).

Lemma 4.2. Define

$$
\psi(x, t)=\frac{k}{2}(x-z)^{2}+\eta(t)
$$

with $k \geq 0, z \in \bar{\Omega}$ and $\eta \in C^{2}([0, T])$. If $U^{*}(x, t)-\psi(x, t)$ has a global maximum in $\left(x_{0}, t_{0}\right) \in \bar{\Omega} \times(0, T]$ for $t_{0}>0$, then

$$
\eta_{t}\left(x_{0}, t_{0}\right)+H\left(k\left(x_{0}-z\right), x_{0}\right) \leq\left(\left\|\eta_{t t}\right\|+C(1+k \operatorname{diam}(\Omega)+k)\right) \lambda
$$

where $\operatorname{diam}(\Omega)=\sup _{x, y \in \Omega}|x-y|$.

Proof. By assumption,

$$
U\left(x, t_{n}\right) \leq U^{*}\left(x, t_{n}\right) \leq \psi\left(x, t_{n}\right)+U^{*}\left(x_{0}, t_{0}\right)-\psi\left(x_{0}, t_{0}\right) .
$$

We may assume that $\psi\left(x_{0}, t_{0}\right)=U^{*}\left(x_{0}, t_{0}\right)$, since only the derivatives of $\psi$ are relevant. Monotonicity of $S_{b}^{\lambda}$ then implies

$$
S^{\lambda}\left(t_{0}-t_{n}\right) U\left(\cdot, t_{n}\right)(x) \leq S_{b}^{\lambda}\left(t_{0}-t_{n}\right) \psi\left(\cdot, t_{n}\right)(x)+U^{*}\left(x_{0}, t_{0}\right)-\psi\left(x_{0}, t_{0}\right)
$$

with $t_{n}<t_{0} \leq t_{n+1}$. If we take the upper semicontinuous envelope on both sides, and set $x=x_{0}$, this becomes

$$
0 \leq\left[S_{b}^{\lambda}\left(t_{0}-t_{n}\right) \psi\left(\cdot, t_{n}\right)\right]^{*}\left(x_{0}\right)-\psi\left(x_{0}, t_{0}\right),
$$

which can be rewritten as

$$
0 \leq \frac{\left[S_{b}^{\lambda}\left(t_{0}-t_{n}\right) \psi\left(\cdot, t_{n}\right)\right]^{*}\left(x_{0}\right)-\psi\left(x_{0}, t_{n}\right)}{t_{0}-t_{n}}+\frac{\psi\left(x_{0}, t_{n}\right)-\psi\left(x_{0}, t_{0}\right)}{t_{0}-t_{n}},
$$

and hence from consistency (4.7):

$$
0 \leq \frac{\left[S_{b}^{\lambda}\left(t_{0}-t_{n}\right) \psi\left(\cdot, t_{n}\right)\right]^{*}\left(x_{0}\right)-\psi\left(x_{0}, t_{0}\right)}{t_{0}-t_{n}}-\psi_{t}\left(x_{0}, t_{0}\right)-\left\|\psi_{t t}\right\|\left(t_{0}-t_{n}\right)
$$

$$
\leq-H\left(D \psi\left(x_{0}, t_{0}\right), x_{0}\right)-\psi_{t}\left(x_{0}, t_{0}\right)+\left\|\psi_{t t}\right\| \lambda+C\left(1+\|D \psi\|+\left\|D^{2} \psi\right\|\right) \lambda .
$$

Analogously, we have

Lemma 4.3. Let $\theta(x, t)=-\frac{k}{2}(x-z)^{2}+\eta(t)$ with $k \geq 0, z \in \bar{\Omega}$ and $\left.\eta \in C^{2}[0, T]\right)$, and let $U_{*}(y, s)-\theta(y, s)$ have a global minimum in $\left(y_{0}, s_{0}\right) \in \bar{\Omega} \times(0, T]$. Assume also $U\left(y_{0}, s_{0}\right)<g\left(y_{0}\right)$ if $y_{0} \in \partial \Omega$. Then

$$
\theta_{s}\left(y_{0}, s_{0}\right)+H\left(D \theta\left(y_{0}, s_{0}\right), y_{0}\right) \geq-\left(\left\|\theta_{s s}\right\|+C\left(1+\|D \theta\|+\left\|D^{2} \theta\right\|\right)\right) \lambda .
$$


Note that in the case $y_{0} \in \partial \Omega$ and $U\left(y_{0}, s_{0}\right)=g\left(y_{0}\right)$, this fails to hold. However, then the Lipschitz type estimate (4.22) given below is sufficient for our purpose.

Proof. Following the arguments (4.14)-(4.17) (note that we have $U\left(x, t_{n}\right) \geq \theta\left(x, t_{n}\right)$ ), we get

$$
\begin{aligned}
0 \geq & -H\left(D \theta\left(0, s_{0}\right), 0\right)-\theta_{s}\left(0, s_{0}\right) \\
& -\left\|\theta_{s s}\right\|\left(s_{0}-s_{n}\right)-C\left(1+\|D \theta\|+\left\|D^{2} \theta\right\|\right) \lambda .
\end{aligned}
$$

For $y_{0} \in \partial \Omega$, use (4.7) instead of (4.5).

Before proving Theorem 4.1, we need some regularity estimates on $U$ at the boundary and initially:

Proposition 4.4. Let $U$ be defined by (4.1)-(4.2). Then, for all $(x, t) \in \bar{\Omega} \times[0, T]$ and $y \in \partial \Omega$ :

$$
\begin{aligned}
|U(x, t)| & \leq\left\|u_{0}\right\|+C_{0} t \\
\left|U(x, t)-u_{0}(x)\right| & \leq C_{S}\left(L_{0}\right) t \\
U^{*}(x, t) & \leq g(y)+\tilde{L}_{S}|x-y|,
\end{aligned}
$$

where $\tilde{L}_{S} \in[0, \infty)$ is given by $L_{0}, L_{S}$ and $T$.

Proof. We first prove (4.21). The proof of (4.20) is similar and easier. Clearly this holds for $t \leq \Delta t$. Assume for induction that

$$
U\left(x, t_{n}\right) \geq u_{0}(x)-C_{S}\left(L_{0}\right) t_{n} .
$$

Then, for $t_{n}<t \leq t_{n+1}$

$$
\begin{aligned}
U(x, t) & =S^{\lambda}\left(t-t_{n}\right) U\left(\cdot, t_{n}\right)(x) \geq S^{\lambda}\left(t-t_{n}\right)\left[u_{0}-C_{S}\left(L_{0}\right) t_{n}\right](x) \\
& \geq u_{0}(x)-C_{S}\left(L_{0}\right) t
\end{aligned}
$$

since $\left\|D\left(u_{0}-C_{S}\left(L_{0}\right) t_{n}\right)\right\| \leq L_{0}$. To prove the opposite inequality, assume that $U\left(x, t_{n}\right) \leq u_{0}(x)+C_{S}\left(L_{0}\right) t_{n}$. Now, $S^{\lambda}$ applied to the right hand side is not defined since $\left.\left(u_{0}+C_{S}\left(L_{0}\right) t_{n}\right)\right|_{\partial \Omega}>g$. However, taking $\bar{g}=g+C_{S}\left(L_{S}\right) T$, we can use (4.8) to get,

$$
\begin{aligned}
U(x, t) & =S^{\lambda}\left(t-t_{n}\right) U\left(\cdot, t_{n}\right)(x) \leq S_{\bar{g}}^{\lambda}\left(t-t_{n}\right) U\left(\cdot, t_{n}\right)(x) \\
& \leq S_{\bar{g}}^{\lambda}\left(t-t_{n}\right)\left[u_{0}+C_{S}\left(L_{0}\right) t_{n}\right](x) \leq u_{0}(x)+C_{S}\left(L_{0}\right) t, \quad t_{n}<t \leq t_{n+1},
\end{aligned}
$$

which is the desired estimate.

To prove (4.22) we start with the inequality

$$
u_{0}(x, t) \leq k|x-y|+g(y)
$$

where $k \geq L_{S} \geq L_{0}$. If (4.9) holds, we get that

$$
S^{\lambda}(\rho) u_{0}(x, t) \leq S_{\bar{g}}^{\lambda}(\rho) u_{0}(x, t) \leq S_{\bar{g}}^{\lambda}(\rho)(k|x-y|+g(y)) \leq k|x-y|+g(y),
$$

where $\bar{g}$ denotes $k|x-y|+g(y)$ restricted to $\partial \Omega$. It hence follows by induction that

$$
U^{*}(x, t) \leq g(y)+L_{S}|x-y| .
$$

If on the other hand the assumption (4.10) holds, we get that

$$
\|D U\| \leq\left(L_{0}+1\right) \exp L_{S} t-1 \text {. }
$$


To see this, observe that (4.10) applied inductively constitutes an Euler approximation to the ordinary differential equation $\left\|U_{x}\right\|_{t}=L_{S}\left(1+\left\|U_{x}\right\|\right)$. From this the result holds with

$$
\tilde{L}_{S}=\left(L_{0}+1\right) \exp L_{S} T-1 .
$$

4.1. Proof of Theorem 4.1. For completeness, we include the whole doubling of variables-argument, although most of it is standard procedure due to Lemmas 4.2 and 4.3. The exception comes when we have a supersolution assuming the boundary condition strongly, in which case we use the regularity of $u$ or (4.22).

We want to estimate $U(x, t)-u(x, t)$ from above first, so we define

$$
M=\sup \left(U^{*}(x, t)-u(x, t)\right),
$$

and assume $M>0$. From here we follow the doubling of variables strategy as in [8] or [17]. Let

$$
\Psi(x, t, y, s)=U^{*}(x, t)-u(y, s)-\frac{(x-y)^{2}}{2 \epsilon^{2}}-\frac{(t-s)^{2}}{2 \epsilon^{2}}-\frac{t+s}{4 T} M .
$$

By upper semicontinuity $\Psi$ attains a maximum in $(\bar{\Omega} \times[0, T])^{2}$. Let

$$
\left(x_{0}, t_{0}, y_{0}, s_{0}\right) \in(\bar{\Omega} \times[0, T])^{2}
$$

be this maximum point.

Then we have

$$
\begin{aligned}
U^{*}\left(x_{0}, t_{0}\right) & -u\left(y_{0}, s_{0}\right)-\frac{\left(x_{0}-y_{0}\right)^{2}}{2 \epsilon^{2}} \\
& \geq \Psi\left(x_{0}, t_{0}, y_{0}, s_{0}\right) \geq \Psi(x, t, x, t) \geq U^{*}(x, t)-u(x, t)-\frac{M}{2} .
\end{aligned}
$$

Taking the supremum of the last expression, we see that

$$
U^{*}\left(x_{0}, t_{0}\right)-u\left(y_{0}, s_{0}\right) \geq M / 2 .
$$

Using the Lipschitz continuity of $u$, we can show that

$$
\left|x_{0}-y_{0}\right| \leq 2 L \epsilon^{2}, \quad\left|t_{0}-s_{0}\right| \leq\left(L_{t}+\frac{R}{2 T}\right) \epsilon^{2}
$$

with $R=\left\|u_{0}\right\|+\max \left(C_{0}, \tilde{C}_{0}\right) T$. Clearly, $\left|\frac{t_{0}-s_{0}}{2 \epsilon^{2}}-\frac{M}{4 T}\right| \leq L_{t}$ by (3.1), hence the last estimate follows from $M \leq 2 R$. To see the first, note that

$$
u\left(y_{0}, s_{0}\right)+\frac{\left(x_{0}-y_{0}\right)^{2}}{2 \epsilon^{2}} \leq u\left(x_{0}, s_{0}\right)
$$

which gives

$$
\frac{\left(x_{0}-y_{0}\right)^{2}}{2 \epsilon^{2}} \leq u\left(x_{0}, s_{0}\right)-u\left(y_{0}, s_{0}\right) \leq L\left|x_{0}-y_{0}\right| .
$$

In the following let

$$
\begin{aligned}
& \theta(y, s)=-\frac{\left(x_{0}-y\right)^{2}}{2 \epsilon^{2}}-\frac{\left(t_{0}-s\right)^{2}}{2 \epsilon^{2}}-\frac{t_{0}+s}{4 T} M \\
& \psi(x, t)=\frac{\left(x-y_{0}\right)^{2}}{2 \epsilon^{2}}+\frac{\left(t-s_{0}\right)^{2}}{2 \epsilon^{2}}+\frac{t+s_{0}}{4 T} M
\end{aligned}
$$


and observe that

$$
D \theta\left(y_{0}, s_{0}\right)=D \psi\left(x_{0}, t_{0}\right) \quad \text { and } \quad\left|D \psi\left(x_{0}, t_{0}\right)\right| \leq 2 L \text {. }
$$

Since $u$ is a supersolution, and $u-\theta$ takes its minimum at $\left(y_{0}, s_{0}\right)$,

$$
\theta_{s}\left(y_{0}, s_{0}\right)+H\left(\theta\left(y_{0}, s_{0}\right), y_{0}\right) \geq 0
$$

unless $y_{0} \in \partial \Omega$ and $u\left(y_{0}, s_{0}\right)=g\left(y_{0}\right)$. Lemma 4.2 applies for $U^{*}-\psi$, so

$$
\begin{aligned}
\frac{M}{2 T} & =\psi_{t}\left(x_{0}, t_{0}\right)-\theta_{t}\left(y_{0}, t_{0}\right) \\
& \leq-H\left(D \psi\left(x_{0}, t_{0}\right), x_{0}\right)+H\left(D \theta\left(y_{0}, s_{0}\right), y_{0}\right)+\left(\frac{1}{\epsilon^{2}}+C\left(1+\frac{\operatorname{diam}(\Omega)}{\epsilon^{2}}+\frac{1}{\epsilon^{2}}\right)\right) \lambda \\
& \leq C_{2 L}\left|x_{0}-y_{0}\right|+\left(\frac{1}{\epsilon^{2}}+C\left(1+\frac{\operatorname{diam}(\Omega)}{\epsilon^{2}}+\frac{1}{\epsilon^{2}}\right)\right) \lambda
\end{aligned}
$$

$$
\leq C_{2 L} 2 L \epsilon^{2}+\frac{1+C\left(1+\operatorname{diam}(\Omega)+\epsilon^{2}\right)}{\epsilon^{2}} \lambda
$$

whenever $t_{0}, s_{0}>0$.

If $t_{0}=0$ or $s_{0}=0$ we may use (3.1), (4.21), (4.34) and (4.35) to prove

$$
M \leq\left(C_{S}\left(L_{0}\right)\left(L_{t}+\frac{R}{T}\right)+2 L_{0} L\right) \epsilon^{2} \quad \text { or } \quad M \leq\left(L_{t}\left(L_{t}+\frac{R}{T}\right)+2 L_{0} L\right) \epsilon^{2} .
$$

We consider $t_{0} \geq 0$ and $s_{0}=0$, which gives the first inequality of (4.42). The second is similar. From (4.34) we have

$$
M / 2 \leq U^{*}\left(x_{0}, t_{0}\right)-u\left(y_{0}, 0\right)=U^{*}\left(x_{0}, t_{0}\right)-u_{0}\left(y_{0}\right) .
$$

Hence

$$
M / 2 \leq\left|U^{*}\left(x_{0}, t_{0}\right)-u_{0}\left(x_{0}\right)\right|+\left|u_{0}\left(x_{0}\right)-u_{0}\left(y_{0}\right)\right| \leq C_{S}\left(L_{0}\right) t_{0}+L_{0}\left|x_{0}-y_{0}\right|,
$$

which implies the first inequality by (4.35).

It remains to consider the case $y_{0} \in \partial \Omega$ and $u\left(y_{0}, s_{0}\right)=g\left(y_{0}\right)=u_{0}\left(y_{0}\right)$. From (4.34), (4.22) and (4.35) we have

$$
\begin{aligned}
M / 2 & \leq U^{*}\left(x_{0}, t_{0}\right)-u\left(y_{0}, s_{0}\right) \leq u_{0}\left(y_{0}\right)+\tilde{L}_{S}\left|x_{0}-y_{0}\right|-u_{0}\left(y_{0}\right) \\
& \leq 2 \tilde{L}_{S} L \epsilon^{2} .
\end{aligned}
$$

Putting these estimates together, and setting $\epsilon=\lambda^{1 / 4}$ gives

$$
M \leq K\left(\left\|u_{0}\right\|, L_{0}, L, L_{S}, C, C_{0}, \tilde{C}_{0}, C_{2 L}, L_{t}, C_{S}\left(L_{0}\right), T\right)\left(\lambda^{\frac{1}{2}}+\lambda\right)
$$

To estimate $U-u$ from below we consider

$$
\Psi(x, t, y, s)=u(x, t)-U_{*}(y, s)-\frac{(x-y)^{2}}{2 \epsilon^{2}}-\frac{(t-s)^{2}}{2 \epsilon^{2}}-\frac{t+s}{4 T} M
$$

and use essentially the same argument. 
5. Example. The proposed abstract scheme is not of much value if a numerically (or theoretically) useful one does not exist. In this section we will develop a Godunov type scheme for an important example.

We will make the additional assumption on $H$ that there exists $M_{R}>0$ such that

$$
|H(p, x)-H(q, x)| \leq M_{R}|p-q| \quad \forall x \in \bar{\Omega},\left\{p, q \in R^{n}:|p|,|q| \leq R\right\} .
$$

Also, we replace Assumption 1 with the existence of a $C_{H} \geq 0$ such that

$$
|H(p, x)-H(p, y)| \leq C_{H}(1+|p|)|x-y|,
$$

however we only use this for deriving a global bound on $|D U|$. Several monotone schemes for the Cauchy problem are given in [15]. Among them the Godunov scheme seems the most convenient to generalize to boundary problems.

5.1. One spatial dimension. Let us first concentrate on the one-dimensional case $\Omega=(0,1), g(x)=0$. The mapping $p \rightarrow H(p, x)$ is assumed to have a unique local minimum at $p=0$ for all $x$. The Godunov scheme $\hat{S}^{h}(\rho)$ is defined here by

$$
\hat{S}^{h}(\rho) u(x)=u(x)-\rho \hat{H}\left(\delta^{-} u(x), \delta^{+} u(x), x\right)
$$

for $h \leq x \leq 1-h$, where

$$
\hat{H}(\alpha, \beta, x)=\left\{\begin{array}{l}
\min _{\alpha \leq p \leq \beta} H(p, x), \alpha \leq \beta \\
\max _{\beta \leq p \leq \alpha} H(p, x), \beta \leq \alpha
\end{array}\right.
$$

and we have employed the one sided difference operators

$$
\delta^{-} u(x)=\frac{u(x)-u(x-h)}{h}, \quad \delta^{+} u(x)=\frac{u(x+h)-u(x)}{h} .
$$

More explicitly, for the Hamiltonian we study

$$
\hat{H}(\alpha, \beta, x)=\left\{\begin{array}{l}
H(\alpha, x), \quad \alpha, \beta \geq 0 \\
H(\beta, x), \quad \alpha, \beta>0 \\
H(0, x), \quad \alpha<0<\beta \\
\max (H(\alpha, x), H(\beta, x)), \quad \beta<0<\alpha .
\end{array}\right.
$$

At $x=0$, we take

$$
\hat{S}^{h}(\rho) u(0)=\min \left(u(0)-\rho H^{-}\left(\delta^{+} u(0), 0\right), 0\right)
$$

where $H^{-}(p, x)=H(\min (0, p), x)=\hat{H}(0, p, x)$. At $x=1$ we similarly take

$$
\hat{S}^{h}(\rho) u(1)=\min \left(u(1)-\rho H^{+}\left(\delta^{-} u(1), 1\right), 0\right)
$$

where $H^{+}(p, x)=H(\max (0, p), x)=\hat{H}(p, 0, x)$.

In order to use the convergence theory, we need to define $\hat{S}^{h}$ for all $0 \leq x \leq 1$. We will do this by extending $u$ to $[-h, 1+h]$, and then apply the interior operator given by (5.3), from now on denoted by $\hat{S}_{0}^{h}$. We consider only $x=0$, the case $x=1$ is entirely similar.

We let $u(x)=u(0)$ for $-h \leq x<0$. This choice implies that $\hat{S}_{0}^{h}(\rho) u(0)=$ $\hat{S}^{h}(\rho) u(0)$ whenever $\hat{S}^{h}(\rho) u(0)<0$. The scheme $\hat{S}_{0}^{h}$ then satisfies all conditions of 
Theorem 4.1 except (4.6) and (4.9). In order to take the boundary condition into account, we take for $0 \leq x<h$

$$
\hat{S}^{h}(\rho) u(x)=\min \left(\hat{S}_{0}^{h}(\rho) u(x), \hat{L}_{S} x\right), \quad \tilde{L}_{S}=L_{0}=\max \left(\left\|\left(u_{0}\right)_{x}\right\|, p_{0}\right) .
$$

If $\hat{S}_{0}^{h}(\rho) u(x)>\hat{L}_{S} x$, we can use that $\hat{L}_{S} x$ is a supersolution to (1.1). We can let $B_{g}$ consist only of functions $u(x) \leq U_{S}$ with $U_{S}(x)=\hat{L}_{S} \min (x, 1-x)$, since $u_{0}(x) \leq U_{S}$, and $\hat{S}^{h}(\rho) U_{S} \leq U_{S}$.

Proposition 5.1. The approximation scheme $\hat{S}^{h}: C^{0,1}([0,1]) \rightarrow C^{0,1}$, satisfies the conditions of Theorem 4.1 with $\lambda=h, C_{S}(L)=\sup _{|p| \leq L, x \in \Omega}|H(p, x)|, \tilde{C}_{0}=$ $C_{0}, L_{S}=L_{0}$ provided

$$
\Delta t M_{R} \leq h, \quad \text { for } \quad R=\left\|u_{x}\right\|
$$

Furthermore

$$
\left\|\left(\hat{S}^{h} u\right)_{x}\right\| \leq \max \left(\left\|u_{x}\right\|, L_{0}\right)+C_{H}\left(1+\max \left(\left\|u_{x}\right\|, L_{0}\right)\right) \Delta t .
$$

Proof. This result is standard except for the Lipschitz continuity (5.11). For that we need

\section{Lemma 5.2. The Godunov Hamiltonian $\hat{H}$ satisfies}

$$
|\hat{H}(\alpha, \beta, y)-\hat{H}(\alpha, \beta, x)| \leq C_{H}(1+\max (|\alpha|,|\beta|)|y-x|
$$

This follows easily from (5.4). Using our extension of $u$ to $[-h, 1+h]$, we first find a bound $\left\|\left(\hat{S}_{0}^{h} u\right)_{x}\right\|$ for the interior operator $\hat{S}_{0}^{h}$ for all $x \in[0,1]$. Define the translation operator $\tau_{\delta}$ by $\tau_{\delta} u(x)=u(x+\delta)$. By Lemma 5.2

$$
\left|\tau_{\delta} \hat{S}_{0}^{h} u(x)-\hat{S}_{0}^{h} \tau_{\delta} u(x)\right| \leq C_{H}\left(1+\max \left(\left\|u_{x}\right\|, p_{0}\right)\right) \Delta t \delta
$$

whenever $x, x+\delta \in[0,1]$. Also $\hat{S}_{0}^{h}$ commutes with addition of constants $\left(\hat{S}_{0}^{h}(u+k)=\right.$ $\left.\hat{S}_{0}^{h}(u)+k\right)$, which from $u \leq v+\|u-v\|$ and monotonicity implies $\left\|\hat{S}_{0}^{h} u-\hat{S}_{0}^{h} v\right\| \leq\|u-v\|$. We can then conclude

$$
\begin{aligned}
\left|\hat{S}_{0}^{h} u(x+\delta)-\hat{S}_{0}^{h} u(x)\right| & \leq\left|\hat{S}_{0}^{h} \tau_{\delta} u(x)-\hat{S}_{0}^{h} u(x)\right|+\left|\tau_{\delta} \hat{S}_{0}^{h} u(x)-\hat{S}_{0}^{h} \tau_{\delta} u(x)\right| \\
& \leq\|u(x+\delta)+u(x)\|+C_{H}\left(1+\max \left(\left\|u_{x}\right\|, p_{0}\right)\right) \Delta t \delta .
\end{aligned}
$$

The estimate (5.11) then follows immediately.

As these considerations demonstrate, finding a scheme with the right properties in the vicinity of the boundary is a theoretical, but not computational, problem. For the analysis, we just need to know that a monotone and consistent scheme exists everywhere.

We conclude as follows

Proposition 5.3. Let $\Omega=(0,1)$, and let the mapping $p \rightarrow H(p, x)$ have a unique minimum at $p=0$ for all $x$. Then (1.1)-(1.2) has a unique viscosity solution $u$ in $C^{0}(\bar{\Omega} \times[0, T])$. Furthermore the scheme $\hat{S}^{h}$ defines an approximation $U(x, t)$ such that $\|U-u\| \leq O(\sqrt{h})$ if the CFL-condition $\Delta t M_{R} \leq h$ holds with

$$
R=\left\|U_{x}(\cdot, t)\right\| \leq\left(L_{0}+1\right) \exp C_{H} t-1 .
$$

The estimate on (5.15), $U_{x}$ follows from (5.11). Observe that (5.11) applied inductively constitutes an Euler approximation to the ordinary differential equation

$$
\max \left(\left\|U_{x}\right\|, L_{0}\right)_{t}=C_{H}\left(1+\max \left(\left\|U_{x}\right\|, L_{0}\right)\right) .
$$


5.2. Two spatial dimensions. We will try to produce a two dimensional example scheme using the same strategy. Consider now the spatial variables $(x, y) \in$ $\Omega=(0,1)^{2}$, and a Hamiltonian

$$
H\left(u_{x}, u_{y}\right)=h\left(u_{x}^{2}, u_{y}^{2}\right)
$$

where $h$ is increasing in both variables. Assumption 4 then clearly holds. As boundary condition we consider $g(x)=0$. The Godunov scheme may be generalized to

$$
\hat{S}^{h}(\rho) u=u-\rho \hat{H}\left(\delta_{x}^{-} u, \delta_{x}^{+} u, \delta_{y}^{-} u, \delta_{y}^{+} u\right)
$$

with

$$
\hat{H}\left(p_{-}, p_{+}, q_{-}, q_{+}\right)=\left\{\begin{array} { l l } 
{ \operatorname { m i n } _ { q _ { - } \leq q \leq q _ { + } } } \\
{ \operatorname { m a x } _ { q _ { + } \leq q \leq q _ { - } } }
\end{array} \left\{\begin{array}{ll}
\min _{p_{-} \leq p \leq p_{+}} H(p, q), & p_{-} \leq p_{+} \\
\max _{p_{+} \leq p \leq p_{-}} H(p, q), & p_{+} \leq p_{-} \\
\min _{p_{-} \leq p \leq p_{+}} H(p, q), & p_{-} \leq p_{+} \\
\max _{p_{+} \leq p_{-} \leq p_{-}} H(p, q), & p_{+} \leq p_{-}
\end{array}, q_{+} \leq q_{+}\right.\right.
$$

For the $H$ given here, this becomes

$$
\hat{H}\left(\left(p_{-}, p_{+}, q_{-}, q_{+}\right)=H(p, q)\right.
$$

where

$$
p=\left\{\begin{array}{l}
p_{+}, \quad p_{-}, p_{+} \leq 0 \\
p_{-}, \quad p_{-}, p_{+} \geq 0 \\
0, \quad p_{-}<0<p_{+} \\
\max \left(p_{-}, p_{+}\right), \quad p_{+}<0<p_{-}
\end{array} \quad q=\left\{\begin{array}{l}
q_{+}, \quad q_{-}, q_{+} \leq 0 \\
q_{-}, \quad q_{-}, q_{+} \geq 0 \\
0, \quad q_{-}<0<q_{+} \\
\max \left(q_{-}, q_{+}\right), \quad q_{+}<0<q_{-}
\end{array}\right.\right.
$$

At the boundaries we may simply replace the undefined finite differences with 0 in $\hat{H}$. That is

$$
\hat{S}_{b}^{h}(\rho) u=u-\rho \begin{cases}\hat{H}\left(0, \delta_{x}^{+} u, \delta_{y}^{-} u, \delta_{y}^{+} u\right), & x=0,0<y<1 \\ \hat{H}\left(\delta_{x}^{-} u, 0, \delta_{y}^{-} u, \delta_{y}^{+} u\right), & x=1,0<y<1 \\ \hat{H}\left(\delta_{x}^{-} u, \delta_{x}^{+} u, 0, \delta_{y}^{+} u\right), & y=0,0<x<1 \\ \hat{H}\left(\delta_{x}^{-} u, \delta_{x}^{+} u, \delta_{y}^{-} u, 0\right), & y=1,0<x<1\end{cases}
$$

and take

$$
\hat{S}^{h}=\min \left(0, \hat{S}_{b}^{h}\right) .
$$

At the corners we have even less information, so we take

$$
\hat{S}_{b}^{h}(\rho) u=u-\rho \begin{cases}\hat{H}\left(0, \delta_{x}^{+} u, 0, \delta_{y}^{+} u\right), & x=0, y=0 \\ \hat{H}\left(\delta_{x}^{-} u, 0,0, \delta_{y}^{+} u\right), & x=1, y=0 \\ \hat{H}\left(\delta_{x}^{-} u, 0, \delta_{y}^{-} u, 0\right), & x=1, y=1 \\ \hat{H}\left(0, \delta_{x}^{+} u, \delta_{y}^{-} u, 0\right), & x=0, y=1 .\end{cases}
$$

This defines an approximation for $(x, y) \in[h, 1-h]^{2} \cup \partial \Omega$. As in one dimension we need to do some work near the boundary. However our strategy there carries over straightforwardly. 
Given a function $u(x, y)$ defined on $[0,1]^{2}$, we want to extend $u$ to $(x, y) \in[-h, 0) \times$ $[0,1]$. We set $u(x, y)=u(0, y)$ there, and similarly around the rest of $\Omega$, and apply the interior operator $\hat{S}_{0}^{h}$. In order to take the boundary condition into account, we take for $0 \leq x<h$

$$
\hat{S}^{h}(\rho) u(x, y)=\min \left(\hat{S}_{0}^{h}(\rho) u(x, y), \hat{U}_{S}(x, y)\right)
$$

with

$$
\hat{U}_{S}(x, y)=\hat{L}_{S} \min (x, y, 1-x, 1-y), \quad \hat{L}_{S}=L_{0}=\max \left(p_{0}, L_{0}\right) .
$$

which is a supersolution to (1.1)-(1.2). Since $\hat{S}^{h}(\rho) U_{S} \leq U_{S}$, and $u_{0} \leq U_{S}$, we can let $B_{g}$ only consist of functions such that $u \leq U_{S}$. We may use the same method as in one dimension to estimate $\left\|D \hat{S}^{h}(\rho) u\right\|$.

Now we can conclude that the conditions of Theorem 4.1 also holds in this case, hence

Proposition 5.4. Let $\Omega=(0,1)^{2}$ and $H$ be defined by (5.16). Then (1.1)(1.2) has a unique viscosity solution $u$ in $C^{0}(\bar{\Omega} \times[0, T])$. Furthermore the scheme $\hat{S}^{h}$ defines an approximation $U(x, t)$ such that $\|U-u\| \leq O(\sqrt{h})$ if the $C F L$-condition $\Delta t C_{C F L} \leq h$ holds for

$$
C_{C F L}=\max _{|p| \leq\left\|U_{x}\right\|,|q| \leq\left\|U_{y}\right\|}\left(\left|H_{p}(p, q)\right|+\left|H_{q}(p, q)\right|\right) .
$$

with

$$
\max \left(\left\|U_{x}\right\|,\left\|U_{y}\right\|\right) \leq\left(L_{0}+1\right) \exp C_{H} t-1
$$

5.3. Numerical results. This two dimensional scheme was implemented and tested. The domain was still $\Omega=(0,1)^{2}$, while $H$ and the boundary conditions varied. Some effects specific to the Cauchy-Dirichlet problem were noted, but in all cases we found that the error is governed by already well known numerical phenomena.

5.3.1. Elliptic paraboloid. As an illustrative example, we chose $H\left(u_{x}, u_{y}\right)=$ $u_{x}^{2}+\frac{1}{4} u_{y}^{2}-1$ and $u_{0}(x, y)=-2 u_{s}(x)$, where $u_{s}$ is the stationary solution, $u_{s}=$ $\min (x, 1-x, 2 y, 2(1-y))$. Figure 5.1 shows the results with discretization parameters $h=0.01$ and $\Delta t=\frac{h}{5}$. At first $U$ only satisfies the boundary condition weakly, but at $t=1.7$ it has reached the approximate stationary solution. The results look reasonable, and there is no sign that the weak boundary statement adds significantly to the error expected for a pure Cauchy or pure Dirichlet problem.

5.3.2. Piecewise linear $H$. The Hamiltonian $H(p, q)=|p|+|q|-1$ allows simple explicit solutions. Firstly, $u_{s}=\min (x, 1-x, y,(1-y))$ is the stationary solution. Starting with $u_{0}=-u_{s}$, the solution will be $u(x, y, t)=\max \left(u_{0}(x, y), t-0.5\right)$ until $t=0.5$, as shown in figure 5.2 to the left. At most points this is easy to check, but it should be proved for the line segment given by $x=y$ and $x \leq 0.5$. At $x<t-0.5$, it follows by the subdifferential

$$
D^{-} u(x, x, t)=\{(p, q):|p|+|q|=1, q \leq 0, p \leq 0\} .
$$

At $x=y=t-0.5=\hat{x}$, we have

$$
u(\hat{x}, \hat{x}, t)-\psi(\hat{x}, \hat{x}, t) \leq u(\hat{x}+h, \hat{x}+h, t-h)-\psi(\hat{x}+h, \hat{x}+h, t-h),
$$



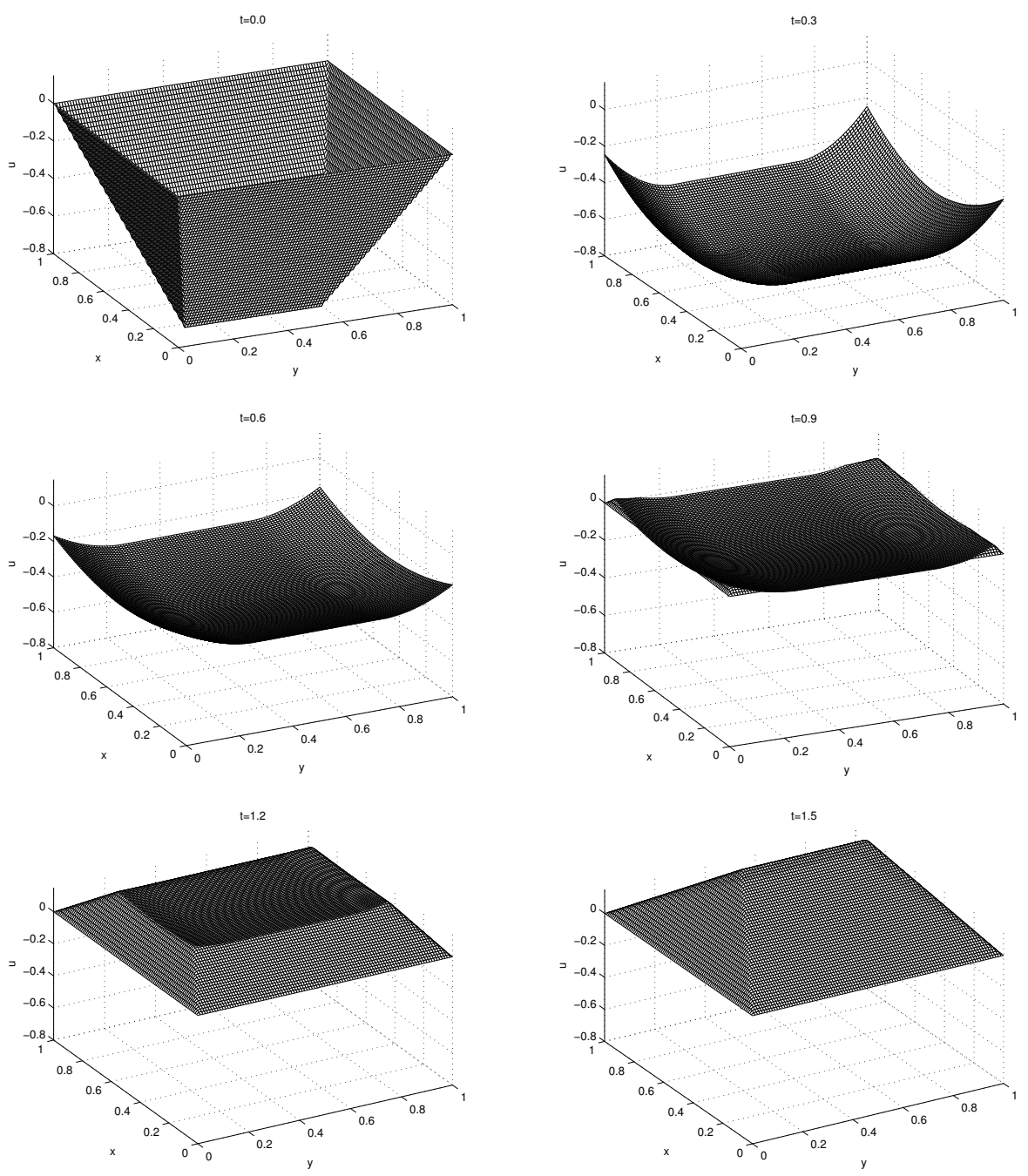

Figure 5.1.

implying

$$
\psi(\hat{x}+h, \hat{x}+h, t-h)-\psi(\hat{x}, \hat{x}, t) \leq-h .
$$

Letting $h \rightarrow 0$, we can conclude that

$$
-\psi_{x}(\hat{x}, t)-\psi_{y}\left(x_{h}, t\right)+\psi_{t}(\hat{x}, t)-1 \geq 0 .
$$

Obviously, $D^{-} u(\hat{x}, \hat{x}, t) \subseteq(-\infty, 0)^{2}$, hence the supersolution condition holds. In the numerical experiments, however, numerical smoothing causes the stationary solution $u_{s}$ to appear prematurely near the corners. On the right in figure 5.2 this is seen for $h=0.02$ and $t=0.01$.

With more grid points, the area where this effect occurs shrinks. Hence, the pointwise error is eventually unaffected by the boundary. Figure 5.3 shows one corner from a simulation with $h=0.0025$ and $\Delta t=h / 2$. These data were simulated for sev- 

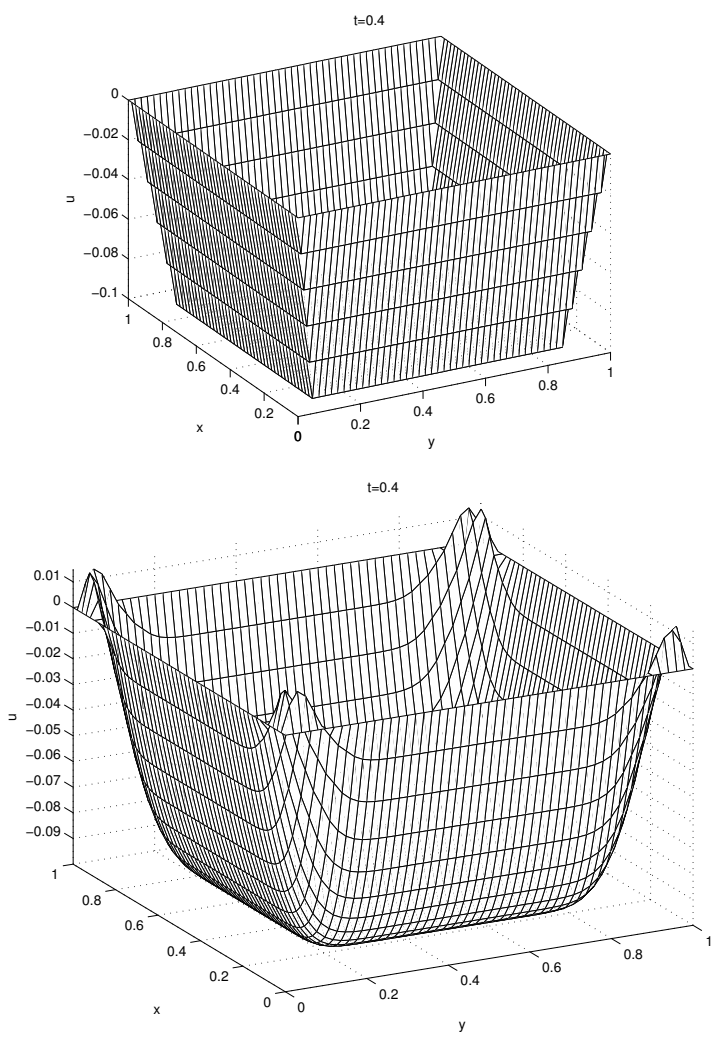

FiguRe 5.2.

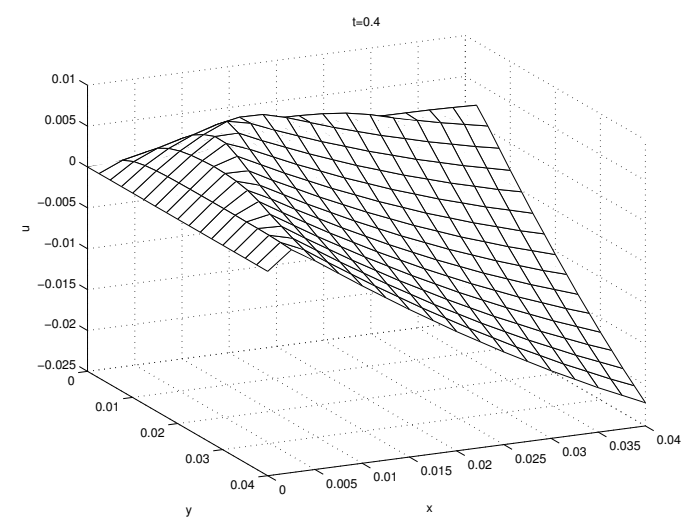

FiguRE 5.3.

eral $h$ (with $\Delta t=h / 2$ ), and the $\log _{2}$-values of the maximal error over the grid points at $t=0.4$ are tabled below. The numbers fit well with the theoretical convergence rate of $\frac{1}{2}$. 


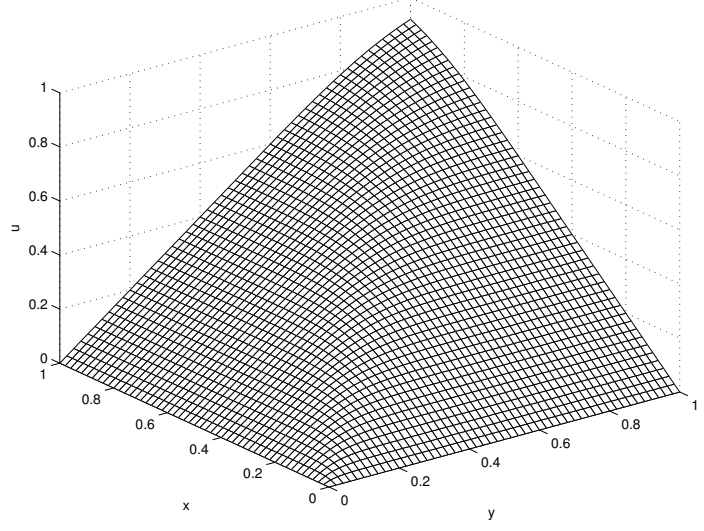

Figure 5.4 .

\begin{tabular}{|l|c|r|}
\hline$h$ & Maximal error over grid & Observed convergence rate \\
\hline 0.04 & 0.0705 & - \\
0.02 & 0.0501 & 0.493 \\
0.01 & 0.0356 & 0.493 \\
0.005 & 0.0252 & 0.498 \\
0.0025 & 0.0178 & 0.502 \\
\hline
\end{tabular}

5.3.3. Numerical diffusion of boundary values. Let $H(p, q)=|p|+|q|-1$ as above. Then $u_{s}=\min (x, y)$ is a stationary solution if also $g(x, y)=\min (x, y)$. Figure 5.4 shows a simulation with $u_{s}$ as initial data. The discretization parameters were $h=0.02$ and $\Delta t=0.01$. Due to numerical smoothing at the line $x=y$, the Dirichlet condition is not strongly satisfied in the discrete case.

\section{REFERENCES}

[1] Rémi Abgrall. Numerical discretization of boundary conditions for first order Hamilton-Jacobi equations. SIAM J. Numer. Anal., 41(6):2233-2261 (electronic), 2003.

[2] Martino Bardi and Italo Capuzzo-Dolcetta. Optimal control and viscosity solutions of Hamilton-Jacobi-Bellman equations. Systems \& Control: Foundations \& Applications. Birkhäuser Boston Inc., Boston, MA, 1997. With appendices by Maurizio Falcone and Pierpaolo Soravia.

[3] G. Barles. Regularity results for first order Hamilton-Jacobi equations. Differential Integral Equations, 3(1):103-125, 1990.

[4] G. Barles. Uniqueness and regularity results for first-order Hamilton-Jacobi equations. Indiana Univ. Math. J., 39(2):443-466, 1990.

[5] G. Barles and B. Perthame. Comparison principle for Dirichlet-type Hamilton-Jacobi equations and singular perturbations of degenerated elliptic equations. Appl. Math. Optim., 21(1):2144, 1990.

[6] G. Barles and P. E. Souganidis. Convergence of approximation schemes for fully nonlinear second order equations. Asymptotic Anal., 4(3):271-283, 1991.

[7] Italo Capuzzo Dolcetta and Fabiana Leoni. On the vanishing viscosity approximation of a time dependent Hamilton-Jacobi equation. In Recent trends in nonlinear analysis, pages 59-75. Birkhäuser, Basel, 2000.

[8] M. G. Crandall and P.-L. Lions. Two approximations of solutions of Hamilton-Jacobi equations. Math. Comp., 43(167):1-19, 1984.

[9] Michael G. Crandall. Viscosity solutions: a primer. In Viscosity solutions and applications (Montecatini Terme, 1995), pages 1-43. Springer, Berlin, 1997.

[10] Michael G. Crandall, Hitoshi Ishii, and Pierre-Louis Lions. User's guide to viscosity solutions 
of second order partial differential equations. Bull. Amer. Math. Soc. (N.S.), 27(1):1-67, 1992.

[11] Michael G. Crandall and Pierre-Louis Lions. Viscosity solutions of Hamilton-Jacobi equations. Trans. Amer. Math. Soc., 277(1):1-42, 1983.

[12] Michael G. Crandall and Richard Newcomb. Viscosity solutions of Hamilton-Jacobi equations at the boundary. Proc. Amer. Math. Soc., 94(2):283-290, 1985.

[13] Wendell H. Fleming and H. Mete Soner. Controlled Markov processes and viscosity solutions, volume 25 of Applications of Mathematics. Springer-Verlag, New York, 1993.

[14] Hitoshi Ishii. A boundary value problem of the Dirichlet type for Hamilton-Jacobi equations. Ann. Scuola Norm. Sup. Pisa Cl. Sci. (4), 16(1):105-135, 1989.

[15] Stanley Osher and Chi-Wang Shu. High-order essentially nonoscillatory schemes for HamiltonJacobi equations. SIAM J. Numer. Anal., 28(4):907-922, 1991.

[16] P. E. Souganidis. A remark about viscosity solutions of Hamilton-Jacobi equations at the boundary. Proc. Amer. Math. Soc., 96(2):323-329, 1986.

[17] Panagiotis E. Souganidis. Approximation schemes for viscosity solutions of Hamilton-Jacobi equations. J. Differential Equations, 59(1):1-43, 1985. 